\title{
Compact Photoacoustic Gas Measuring System for Carbon Dioxide Indoor Monitoring Applications
}

\author{
Alexander Ambs ${ }^{1}$, Jochen Huber ${ }^{1}$, Jürgen Wöllenstein ${ }^{1,2}$ \\ ${ }^{1}$ Fraunhofer Institute for Physical Measurement Techniques (IPM), Heidenhofstr. 8, \\ 79110 Freiburg, Germany \\ alexander.ambs@ipm.fraunhofer.de \\ 2 IMTEK, Department of Microsystems Engineering, Faculty of Applied Sciences, \\ University of Freiburg, Georges-Köhler-Allee 102, 79110 Freiburg, Germany
}

\begin{abstract}
We present a compact photoacoustic gas measuring system for carbon dioxide monitoring in indoor applications. The system components an IR-source, an encapsulated microphone as acoustic sensor and a 3D printed circuit board (PCB) design. The compact 3D electronic design replaces an additional sensor housing. $\mathrm{CO}_{2}$ concentrations from $0 \mathrm{ppm}$ to $5000 \mathrm{ppm} \mathrm{CO} 2$ can be easily detected. The system has a resolution limit of $50 \mathrm{ppm}$ for low $\mathrm{CO}_{2}$ concentrations. Long-term measurements show the reproducibility. With the integrated Pt10000 sensor temperature compensation is possible. The absorbing gas concentration is measured directly by detecting pressure changes inside a closed reference chamber.
\end{abstract}

Key words: photoacoustics, carbon dioxide, gas sensor, IR absorption, indoor air quality

\section{Introduction}

Indoor air quality monitoring is important in work environment and educational facilities. Especially in open-plan offices and class rooms, there are a lot of persons within a small area which results in a rapid deterioration of indoor air quality. $\mathrm{CO}_{2}$ concentration correlates with the number of persons inside a room and is next to humidity and temperature the main indicator for indoor air quality. Carbon dioxide at high levels may cause symptoms like feeling tired, getting drowsy or headaches. To ensure that the indoor carbon dioxide level will be less than a critical value of $1000 \mathrm{ppm}$, continuous indoor air quality monitoring and controlled room ventilation is used. Controlled room ventilation improves the working and living conditions and reduces energy consumption. Due to these facts there is a high demand for low-cost, small and energy-efficient gas measuring systems for real-time and selective $\mathrm{CO}_{2}$ measurements in indoor air quality monitoring applications. There are no commercially available systems for indoor carbon dioxide monitoring based on the principle of photoacoustics. Most commercially available measuring systems are based on the principle of a filter photometer. Motivated by these facts, this work aims to develop and characterize a compact gas measuring system for $\mathrm{CO}_{2}$ indoor air quality monitoring applications. The developed carbon dioxide gas measuring system is based on the principle of photoacoustics. The photoacoustic measuring principle depends on selective infrared absorption of infrared-active gases. $\mathrm{CO}_{2}$ strongly absorbs mid-infrared light $\left(\lambda_{\mathrm{CO} 2}=4,3\right.$ $\mu \mathrm{m})$. Absorbed infrared radiation stimulates infrared-active molecules like $\mathrm{CO}_{2}$ to vibrate and rotate. The conversion of absorbed radiation energy into kinetic energy results in a gas expansion. We have a two-chambersystem with a reference chamber filled with a high amount of $\mathrm{CO}_{2}$ and hermetically sealed with an infrared transparent window as well as an acoustic sensor (microphone) placed inside. With a time-modulated radiation source, the gas expansion results in a periodic time-modulated pressure difference inside the closed volume [1]. The microphone detects the alternating pressure difference inside the reference chamber. The concentration of $\mathrm{CO}_{2}$ between the radiation source and the reference chamber affects the radiation intensity which impacts the reference chamber and thus the pressure difference. The alternating pressure difference can be calculated from the absorption strength and the IR-emitter emission profile [2]. The measurement chamber is located between the radiation source and the reference chamber. 


\section{Theory}

Basis for selective infrared radiation absorption is a temporal changing dipole moment of the infrared-active gas. IR-active gases absorb lowenergy infrared radiation at characteristic wavelengths, described by Lambert-Beer-Law $[3,4]$. If electromagnetic radiation energy is equal to the energy difference of nearby energy levels absorption occurs. Ground state electrons are stimulated to an excited state. Infrared radiation absorption cause IR active molecules to vibrate and rotate at molecule specific wavelengths (absorption lines). Inelastic collisions between excited molecules and non-excited ones result in a change of IR radiation energy into kinetic energy. Increased kinetic energy causes a gas expansion and thus a pressure difference in a closed volume. The total absorbed power $P_{\text {tot }}$ is given by the integral over the multiplication of the absorption profile $A(\lambda)$ with the emitted power profile of the infrared emitter $P_{\text {emi }}(\lambda)$. The absorption profile correlates with the transmission profile $T(\lambda)$ as follows:

$$
A(\lambda)=1-T(\lambda)
$$

For further calculations we make assumptions that the measured medium behaves like an ideal gas and there are neither reflections nor heating losses. Therefore a loss of radiation intensity is explained by power absorption of the ideal gas only.

$$
P_{\text {tot }}=\int_{\lambda_{\min }}^{\lambda_{\max }} A(\lambda) \cdot P_{\text {emi }}(\lambda) d \lambda
$$

Fig. 1 shows a simulated transmission profile of $\mathrm{CO}_{2}$ at ambient temperature of $T=25^{\circ} \mathrm{C}$ and pressure of $p=1013$ mbar.

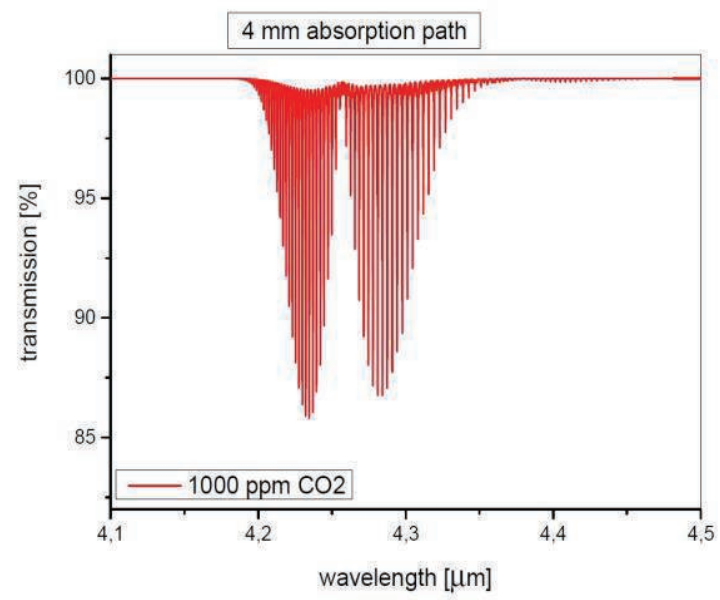

Fig. 1: Line spectrum of 1000 ppm $\quad \mathrm{CO}_{2}$ concentration for an absorption length of $4 \mathrm{~mm}$ [5].
The total absorbed power is stored as thermal energy $Q$ inside the ideal gas volume. With a modulation frequency $f=13 \mathrm{~Hz}$ and a rectangular stimulus with duty cycle $v=0,5$ thermal energy is described in the following equation:

$Q=\frac{P_{t o t} \cdot v}{f}$

The quotient of stored thermal energy $Q$ and gas specific thermal capacity $C_{V}$ results in the temperature difference $\Delta T$ :

$\Delta T=\frac{Q}{C_{V}}$

Using the ideal gas law allows us to determine the pressure difference $\Delta p$ :

$\Delta p=\frac{n \cdot R \cdot T}{V}$

With the described pressure difference a pressure wave inside the closed volume occurs. Its amplitude is a qualitative measure of the absorbed radiation intensity and the absorbing gas concentration.

\section{Measuring system}

Fig. 2 shows schematically the photoacoustic measuring principle. The measuring system (Fig. 3) is set up as a two-chamber-system embedded within a compact 3D electronic design which replaces an additional sensor housing. As infrared source a broadband emitter (IR-50S, HawkEye) in a TO-5 case is used. As acoustic sensor we make use of a commercially available MEMS-microphone (SMM310, Infineon) as it is used in mobile phones. The microphone is placed in a TO-39 case filled with a high amount of carbon dioxide (reference chamber). Both cases are hermetically sealed with an infrared transparent window made of sapphire (emitter) and calcium fluoride (microphone). Both elements are fixed in a 3D PCB design which contains a microcontroller (PSoC5, Cypress) as well as analog electronic circuits. Absorption length and optical path can be varied. The microcontroller appears complete internal integration of analog electronics as well as the control of analog components and digital signal processing. Thus a minimum of analog electronic components is required. Signal processing includes filtering, amplifying and digitalization of the noisy microphone signal. To filter the signal a digital lock-in-amplifier is used. The digital lock-in amplifier is realized in the microcontroller. A lock-in-amplifier is a phase sensitive rectifier 


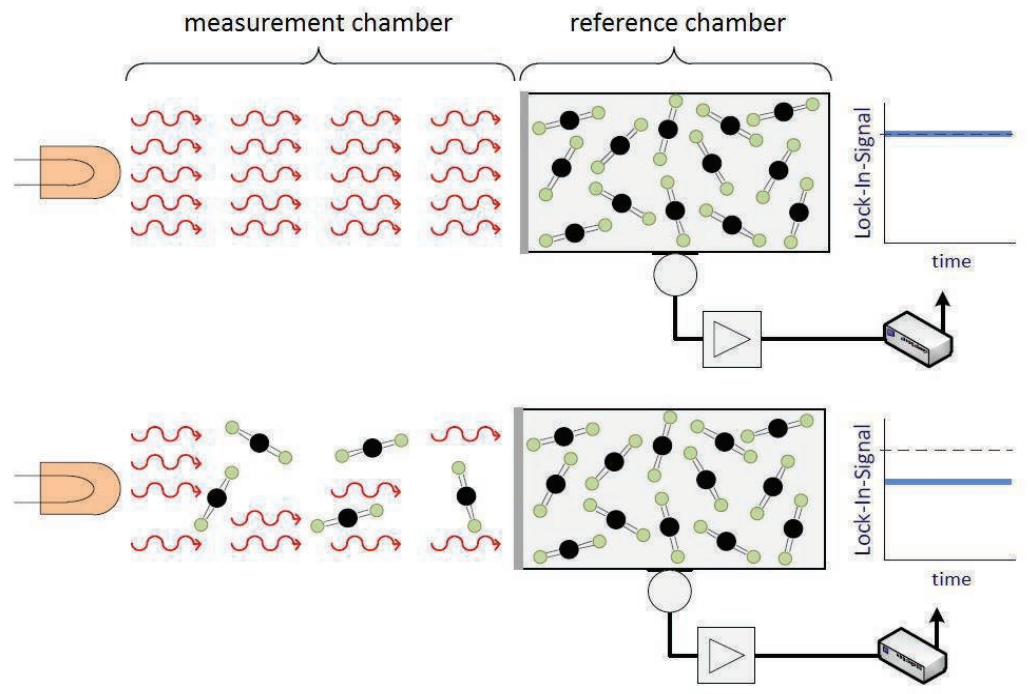

Fig. 2: Schematic representation of photoacoustic signal detection with time-modulated IR-emitter, measurement chamber and reference chamber with acoustic sensor and filled with a high amount of $\mathrm{CO}_{2}$.

and frequency-selective, narrow bandpass filter which helps to get a better signal-to-noise ratio [6]. For lock-in-amplifier internal multiplication a reference signal with exactly same frequency as the alternating, periodic measurement signal is required. The IR-emitter is stimulated by a sinusoidal, time-modulated voltage with defined frequency. This time-modulated signal is realized in the microcontroller and is used as reference signal. A lock-in output signal is given as a digital value which depends on the amplitude of the periodic measurement signal.

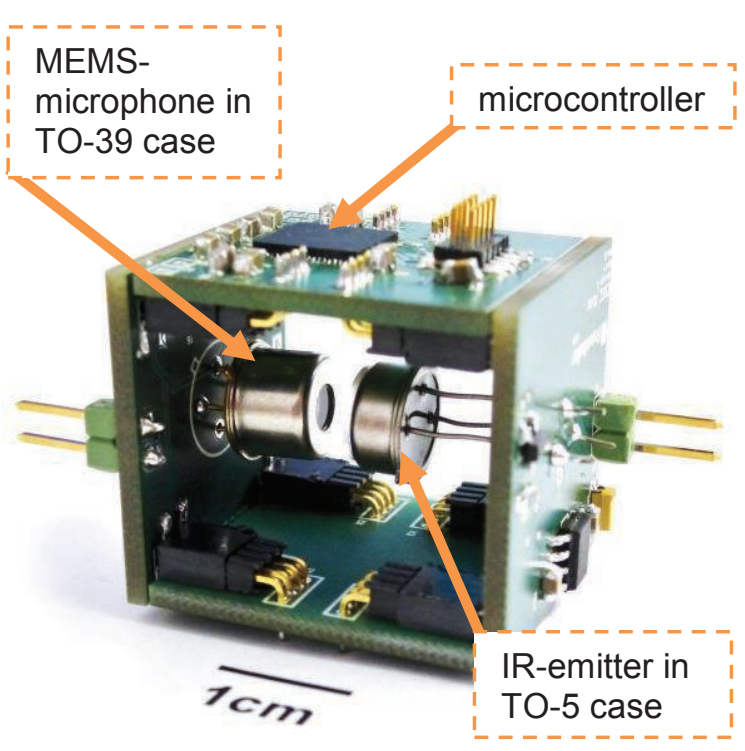

Fig. 3: Measuring system in 3D electronic design contains IR-emitter, MEMS-microphone, PSoC5 and other analog electronic components.

\section{Experimental Results and Discussion}

For following measurements an optical path length of $5 \mathrm{~mm}$ was configured. Nitrogen was used as carrier gas. The signals are represented by digitized microcontroller values which have no unit. Maximum measurement values are given for $100 \%$ nitrogen and 0 ppm $\mathrm{CO}_{2}$ concentration (baseline). Fig. 4 shows a long-term measurement of concentration steps from 200 to $1000 \mathrm{ppm} \mathrm{CO} 2$ concentration with step size of $200 \mathrm{ppm}$. It can be seen that the system has a resolution better than 200 ppm. Further measurements show that a resolution of $50 \mathrm{ppm}$ is possible. After each concentration step the measuring signal returns to a constant and stable baseline. A response time of $60-90$ seconds is given by signal changes between $\mathrm{CO}_{2}$-concentration steps and $100 \%$ nitrogen. The measurement in Fig. 5 shows temperature
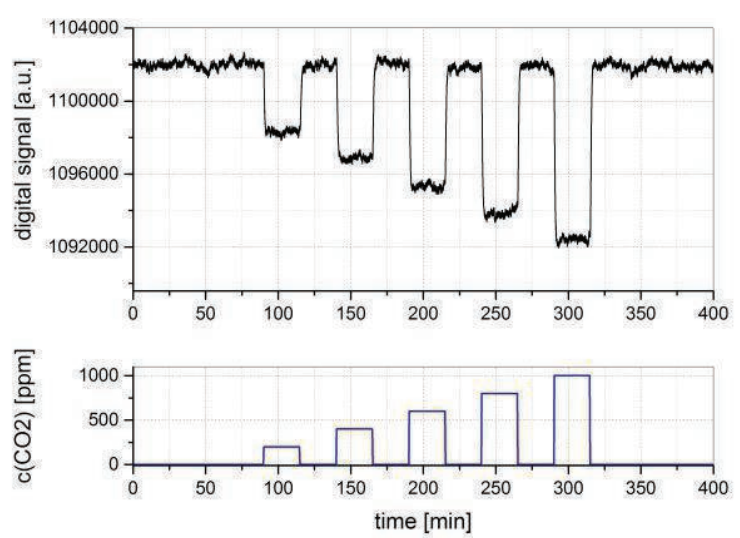

Fig. 4: Measurement of carbon dioxide in nitrogen in a range from 200-1000 ppm and concentration steps of $200 \mathrm{ppm}$. 

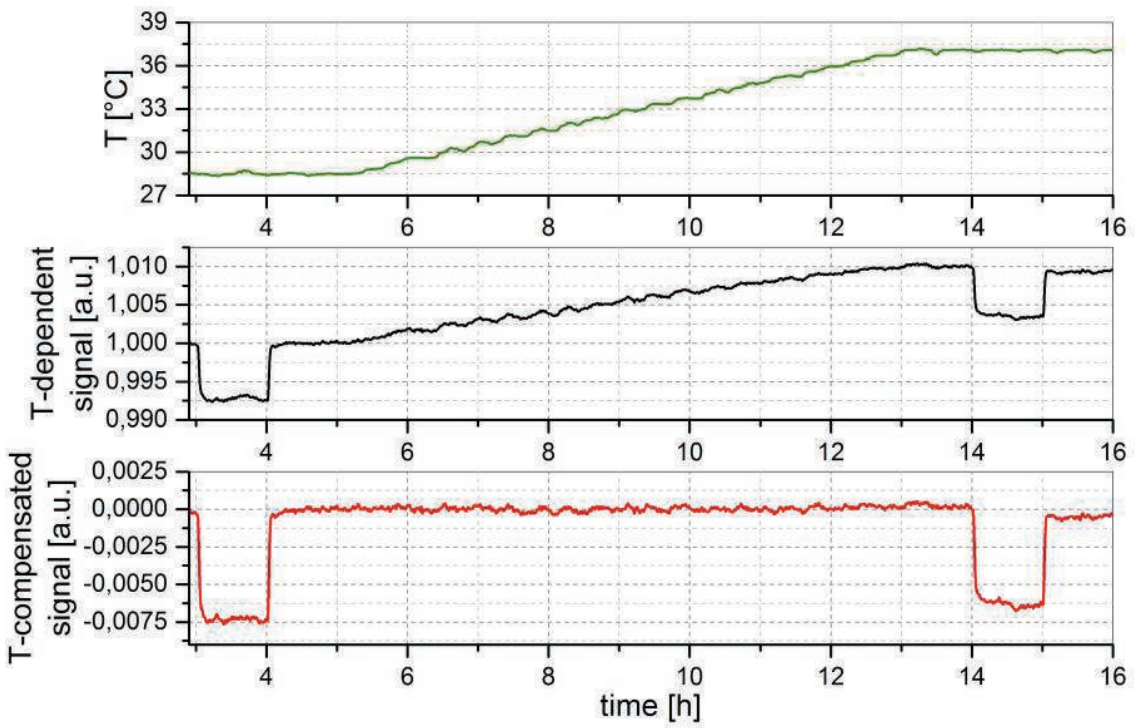

Fig. 5: Top: measured temperature values; middle: measured temperature-dependent values; bottom: temperature compensated values.

compensation with integrated Pt10000 sensor. In a climate chamber the measuring system was exposed to a temperature profile $(\Delta T=10$ $\left.{ }^{\circ} \mathrm{C}\right)$. In this case measured temperature values correspond to the temperature near the system surface and is not equal to the ambient temperature profile. At both temperature plateaus of ambient temperature $\left(15^{\circ} \mathrm{C}\right.$ and 25 ${ }^{\circ} \mathrm{C}$ ) the measuring System was exposed to 1000 ppm CO 2 concentration for 1 hour. Fig. 5 shows that measuring signal changes for both $\mathrm{CO}_{2}$ concentration steps (1000 ppm) at both temperature plateaus vary differently. This is caused by physical effects which are not further discussed in this paper. A polynomial FITfunction was calculated from measured temperature values and standardized temperature-dependent measuring values. The FIT-function allows an acceptable solution of temperature compensation to get a signal independent of temperature.

\section{Conclusion}

The presented compact sensor system can be integrated in public means of transport or classrooms. The special 3D electronic design provides a small sensor size. It can be used for indoor monitoring applications to measure increase of carbon dioxide concentration. Based on the principle of photoacoustics radiation source, acoustic sensor and measured medium are not in direct contact but physically separated. From 0 ppm to 5000 ppm the system has a resolution better than 200 ppm. For low $\mathrm{CO}_{2}$ concentrations the system has a resolution limit of $50 \mathrm{ppm}$. With the integrated Pt10000 sensor temperature compensation is realized. The total signal processing is integrated in a microcontroller. Potential for further miniaturization is given by using SMD components. Furthermore a calibration measurement would allow output values of $\mathrm{CO}_{2}$ concentration in $\mathrm{ppm}$.

\section{References}

[1] Z. Bozóki, A. Pogány, G. Szabó: Photoacoustic Instruments for Practical Application: Present, Potentials and Future Challenges. Applied Spectroscopy Reviews, 46:1-37 (2011)

[2] J. Huber, J. Wöllenstein: Kompaktes photoakustisches Gasmesssystem mit Potential zur weiteren Miniaturisierung. Technisches Messen, Band 80, Heft 12 (2013)

[3] E. D. T. Atkins, P. W. Atkins and J. de Paul: Physikalische Chemie. WILEY-VCH, 5. Auflage (2013)

[4] W. Demtröder: Experimentalphysik 2 Elektrizität und Optik. Springer-Verlag (2008)

[5] Spectralcalc by GATS Inc., Software zur Simulation von Absorptionsspektren. http://www.spectralcalc.com (2014)

[6] W. Schmidt: Optische Spektroskopie - Eine Einführung. WILEY-VCH (2000) 